\title{
Article
}

\section{Eye movements reveal a similar Positivity Effect in Chinese and UK older adults}

Wang, Jingxin, Xie, Fang, He, Liyuan, Meadmore, Katie L., Paterson, Kevin B. and Benson, Valerie

Available at http://clok.uclan.ac.uk/33164/

Wang, Jingxin, Xie, Fang, He, Liyuan, Meadmore, Katie L., Paterson, Kevin B. and Benson, Valerie ORCID: 0000-0002-0351-4563 (2020) Eye movements reveal a similar Positivity Effect in Chinese and UK older adults. Quarterly Journal of Experimental Psychology, 73 (11). pp. 1921-1929. ISSN 1747-0218

It is advisable to refer to the publisher's version if you intend to cite from the work. http://dx.doi.org/10.1177/1747021820935861

For more information about UCLan's research in this area go to http://www.uclan.ac.uk/researchgroups/ and search for <name of research Group>.

For information about Research generally at UCLan please go to http://www.uclan.ac.uk/research/

All outputs in CLoK are protected by Intellectual Property Rights law, including Copyright law. Copyright, IPR and Moral Rights for the works on this site are retained by the individual authors and/or other copyright owners. Terms and conditions for use of this material are defined in the policies page. 
Eye movements reveal a similar Positivity Effect in Chinese and UK older adults

Jingxin Wang ${ }^{1}$

Fang Xie ${ }^{1}$

Liyuan $\mathrm{He}^{1}$

Katie L. Meadmore ${ }^{2}$

Kevin B. Paterson ${ }^{3}$

Valerie Benson ${ }^{4}$

1. Faculty of Psychology, Tianjin Normal University, China.

2. School of Psychology, University of Southampton, UK.

3. Department of Neuroscience Psychology and Behaviour, University of Leicester, UK.

4. School of Psychology, University of Central Lancashire, UK

Author Note: Jingxin Wang and Fang Xie are joint first authors and contributed equally to this work.

Correspondence can be addressed to Jingxin Wang, Academy of Psychology and Behavior, Tianjin Normal University, Hexi District, Tianjin, China, 300374, Email:

wangjingxin@tjnu.edu.cn or Valerie Benson, School of Psychology, University of Central Lancashire, Preston, UK, PR1 2HE. Email: vbenson3@uclan.ac.uk

JW, FX, LH and VB contributed to the design of the experiment. FX and LH implemented 
the experiment. FX, LH and KM collected the data. FX analysed the data. FX, JW, KP and VB wrote the manuscript. 
Abstract

The "positivity effect" (PE) reflects an age-related increase in the preference for

3 positive over negative information in attention and memory. The present experiment

4 investigated whether Chinese and UK participants produce a similar PE. In one experiment

5 we presented pleasant, unpleasant and neutral pictures simultaneously and participants

6 decided which picture they liked or disliked on a third of trials respectively. We recorded

7 participants' eye movements during this task and compared time looking at, and memory for,

8 pictures. The results suggest that older but not younger adults from both China and UK

9 participant groups showed a preference to focus on and remember pleasant pictures,

10 providing evidence of a PE in both cultures. Bayes Factor analysis supported these

11 observations. These findings are consistent with the view that older people preferentially

12 focus on positive emotional information, and that this effect is observed cross-culturally.

14 Keywords: Ageing and emotion bias; Eastern versus Western cultures; Eye tracking methods 
Evidence suggests that as people age they develop a bias to focus on and process

2 positive emotional stimuli (Isaacowitz, Löckenhoff, Lane, et al., 2007). This is known as the

3 Positivity Effect (PE; Carstensen, Isaacowitz, \& Charles, 1999; Isaacowitz, Wadlinger,

4 Goren, \& Wilson, 2006; Fung, Lu, Goren et al., 2008; Kwon, Scheibe, Samanez-larkin, Tsai,

5 \& Carstensen, 2009; Wang, He, Jia, Tian, \& Benson, 2015), and is defined as preferential

6 cognitive processing of positive affective information, along with avoidance of negative

7 affective information in the environment (Kwon et al., 2009).

9 Carstensen, 2012) postulates that with age, people are motivated to derive emotional meaning

10 from life, leading them to pay more attention to positive, relative to negative/neutral,

11 information (Fung et al., 2008; Kwon et al., 2009). Accordingly, older adults should show

12 increased preference for positive emotional information compared to younger adults (Wang

13 et al., 2015). The SST suggests that in older people, the perception of a limited future life-

14 time motivates development of the PE. When future life-time is perceived as less limited, as

15 for young adults, people tend to pursue goals with future benefits (e.g., education,

16 employment). However, when the future appears limited, as for older adults, individuals

17 focus instead on goals that benefit them in the present time (Isaacowitz et al., 2006).

The PE is well-documented in Western cultures, with studies showing that older adults

19 look less at negative and more at positive stimuli compared with young adults (Charles,

20 Mather, \& Carstensen, 2003; Mather \& Carstensen, 2003; Hess, Auman, Colcombe, \&

21 Rahhal, 2003; Isaacowitz et al., 2006; but see Isaacowitz, Livingstone, Harris, \& Marcotte, 
1 2015). For example, Mather and Carstensen (2003) found that older participants responded

2 faster to a dot probe if this was presented on the same side as a neutral face, than when it was

3 presented on the same side as a negative face. Moreover, older participants remembered the

4 positive faces better than the negative faces in that study. Similarly, Hess et al. (2003) tested

5 younger and older participants and found that when participants were primed with negative

6 pictures, memory performance decreased in recall and recognition tasks in comparison to

7 those who were not primed with negative information. In a later study, Isaacowitz and

8 colleagues, found that older participants preferred to look at positive (e.g. happy) faces and

9 avoided attending to negative (e.g. angry, sad) faces (Isaacowitz et al., 2006). Research also

10 reports a shift with age in the ratio of positive versus negative information attended to or

11 remembered (Charles et al., 2003; Chung \& Lin, 2012), with older participants more likely to

12 remember positive information. Moreover, while the degree to which the PE may change

13 with age varies across studies (Charles, Reynolds, \& Gatz, 2001; Mroczek, \& Kolarz, 1998),

14 such effects appear present throughout older adulthood in Western cultures.

In Western cultures the focus is on individual development and achievement of goals,

16 where there is strong encouragement for independence and a public expression of one's

17 achievements. Cultivation of optimism and a sense of pride is promoted at an individual

18 level, and, compared with East Asians living in collective cultures, Western people who

19 believe in individualism, have preferential cognitive processing of positive stimuli with high

20 arousal levels (Tsai, Knutson, Fung, 2006). 
In contrast, in East Asian cultures, the focus is on interdependence between adults,

2 leading to collectivist societies, where group harmony is considered to be optimal.

3 Individualism is not considered to be of main importance in such a culture, and as a result of

4 this it is inevitable that individuals experience concession and endurance to enhance the

5 group goals of unity and solidarity. It has been suggested that such experiences will involve

6 the suppression of some positive emotions in such a culture, perhaps leading to an absence of

7 a PE in East Asian cultures (Wang et al., 2015).

However, the existence of a PE in Eastern cultures is controversial. In an attempted

9 replication of Isaacowitz et al.'s (2006) study, Fung et al. (2008) found no attentional

10 preference for happy over neutral faces in older Hong Kong Chinese participants. They

11 inferred that this null effect provided evidence for a cultural difference between Western and

12 Eastern participants and they proposed that negative and positive information is equally

13 important in Eastern cultures. However, as Fung et al. did not directly compare Western and

14 Eastern participant groups, it is unclear whether their findings necessarily do show this

15 proposed cultural difference. Moreover, alternative research studies have reported that the PE

16 does exist in Eastern populations. For example, using a picture recognition task Kwon et al.

17 (2009) showed enhanced memory for pleasant compared to unpleasant pictures in older

18 compared to younger Korean adults, but they did not compare the effect cross-culturally.

19 More recently, Wang et al. (2015) reported a PE among older, but not younger, Chinese

20 adults. Specifically, younger and older Chinese participants showed an emotional bias for

21 both pleasant and unpleasant pictures when these were paired with a neutral picture, but only 
1 the older adults showed avoidance of negative pictures when these were paired with positive

2 pictures. Again, this study was not conducted cross-culturally which means that a direct

3 comparison cannot be made between Eastern and Western cultures from that study. Thus, an

4 important aim of the present study was to directly compare the PE across Eastern and

5 Western samples.

6 Most previous studies have used techniques in which pleasant and unpleasant pictures

7 are presented separately, alongside a neutral picture, to assess biases towards pleasant

8 pictures in pleasant versus neutral comparisons, and biases away from unpleasant pictures in

9 unpleasant versus neutral comparisons (Isaacowitz et al., 2006; Fung et al., 2008). However,

10 Wang et al. (2015) found the most robust PE when unpleasant and pleasant pictures were

11 presented together. Therefore, we used a variant of this method in the current study, by

12 assessing gaze patterns for pleasant, unpleasant and neutral images that were presented

13 simultaneously. This novel method allows us to investigate the preference to focus on

14 pleasant emotional pictures, along with avoidance of processing unpleasant pictures (as

15 measured by eye-fixations on, and subsequent memory for all types of pictures) when all

16 types of pictures are presented in the same trial. The task we used involved participants

17 making a judgment on a proportion of trials to indicate which was the most or least preferred

18 picture. Asking participants to choose their most or least preferred picture ensured that all

19 pictures presented in the displays were engaged with and fixated during each trial. Hence, the

20 pictures would not be processed mindlessly - as might be the case in an entirely free-viewing

21 paradigm. 
Based on SST, which proposes that individuals who demonstrate a PE prefer to focus

2

on positive stimuli and avoid negative stimuli, we predicted that initial looking time on each image would be longer for pleasant relative to unpleasant pictures in older participants in both cultures. We also predicted that older participants would have better memory for the pleasant pictures.

Ethics Statement. This study was approved by the research ethics committee of the Faculty of Psychology at Tianjin Normal University and University of Southampton, and each participant provided written informed consent.

Participants. The Chinese sample were 24 young adults aged 21-26 years ( $M=24$ years) from Tianjin Normal University, and 24 older adults aged 65-80 years (M=70 years) from the Tianjin community. The UK sample were 24 young adults aged 19-29 years from University of Southampton, UK (M=21 years) and 24 older adults aged 65-89 years ( $\mathrm{M}=74$ years) from the Southampton and Leicester communities. All participants were right-handed, had normal or corrected-to-normal vision (20/30 or better) determined using a Tumbling E acuity chart (Taylor, 1978). All participants reported no neurological or mental disorders, and all showed typical cognition as assessed using the Montreal Cognitive Assessment (Beijing version in China, Original version in UK; applying a standard exclusion criterion of $<26 / 30$; Nasreddine et al., 2005). Participants were selected to have at least 11 years of formal education (equivalent to senior high schooling). No participants had taken part in similar experiments.

Before taking part in the current experiment demographic data and scores on the Positive 
1 Affect and Negative Affect Scale (PANAS) (Watson, Clark, \& Tellegen, 1988, revised and

2 adapted for Chinese by Qiu et al. (2008)) were recorded. There were no differences between

3 Chinese young and older participants in Positive Affect (PANAS) (young adults: $M=28$,

$4 S D=.88$; older adults: $M=27, S D=1.66 ; t(46)=0.64, p=.50)$ or Negative Affect (PANAS)

5 (young adults : $M=12, S D=1$; older adults: $M=11, S D=2 ; t(46)=1.35, p=.187$ ). Similarly,

6 there was no difference for UK young and older participants in Positive Affect (PANAS)

7 (young adults: $M=31, S D=4$; older adults: $M=34, S D=7 ; t(46)=1.82, p=.08$ ), or Negative

8 Affect (PANAS) (young adults: $M=13, S D=3$; older adults: $M=13, S D=3 ; t(46)=0.00$,

$9 p=1.00)$.

10 Vocabulary and short-term memory were assessed using the Wechsler Intelligence Test

11 (Wechsler, 1997). Vocabulary scores were similar across Chinese young and older adults

12 (young adults, $M=61, S D=8$; older adults, $M=59, S D=8 ; t(46)=0.68, p=.500$ ). A similar

13 pattern was also observed for UK older and young adults (young adults, $M=57, S D=5$; older

14 adults, $M=60, S D=5 ; t(46)=1.72, p=.09)$. As is typical for these age-groups (Ryan, Sattler, \&

15 Lopez, 2000), digit span scores were lower for the older than the younger adults in both the

16 China sample (young adults, $M=15, S D=3$; older adults, $M=12, S D=2 ; t(46)=4.77, p<.001$ ),

17 and the UK sample (young adults, $M=18, S D=3$; older adults, $M=17, S D=2 ; t(46)=2.11$,

$18 p=.04)$, and note, all the values of vocabulary and digit span refer to raw scores.

Materials and Design. Picture stimuli were from the International Affective Picture

20 System (IAPS; Lang, Bradley, \& Cuthbert, 2005), rated for valence and arousal by Western

21 young adults. Two hundred and thirty-six pictures were chosen from the database based on 
1 these scores. For the selected pictures, we conducted additional assessments of valence and

2 arousal using groups of Eastern (China) young and older adults, and Western (UK) older

3 adults. Forty-seven young Chinese adults aged 19-26 years ( $M=23$ years), and 44 older

4 Chinese adults aged 60-78 years ( $M=67$ years) and 10 older UK adults aged 66-72 years

5 ( $M=66$ years) re-rated the pictures for valence and arousal using the same scale. The raters

6 did not take part in the experiment. We then compared these scores with scores obtained from

7 the database (IAPS). The results showed strong correlations between the scores rated by each

8 group of raters and the scores from the original USA sample, indicating that our participant

9 groups agreed on the emotionality of the pictures. Correlation coefficients are shown in Table

10 1. From the rated pictures we selected 36 pleasant, 36 unpleasant, and 36 neutral pictures as

11 stimuli. For these stimuli, participants closely agreed on valence and arousal scores, and

12 stimuli were selected so that unpleasant pictures had lower valence but the same arousal as

13 pleasant pictures, and neutral pictures had higher valence than unpleasant pictures, lower

14 valence than pleasant pictures, and lower arousal compared to both. We controlled the

15 luminance levels of the pictures using Adobe Photoshop 7.0. to ensure that this did not differ

16 significantly across the pictures $(p>0.05)$. The means and standard deviations of the

17 luminance level of each picture were computed using Scion Image software (Scion Corp,

18 version Alpha 4.0.3.2, Frederick, Maryland).

Insert Table 1

The pictures were randomly assigned to form 36 displays, each display included a

21 single pleasant, neutral and unpleasant picture. The emotional pictures in each display were 
1 matched on arousal and valence. The three pictures that made up each trial display were

2 presented together only once, and no pictures were presented twice in the same location. A

3 total of four blocks of 36 pictures was created and all participants completed all four blocks.

4 A mixed measures design was used with the within-participant factor picture-type (pleasant,

5 neutral, unpleasant) and between-participant factors age-group (older adults, younger adults)

6 and culture (China, UK). There were 4 display blocks, each containing 36 experimental trials

7 and 2 practice trials.

Apparatus and Procedure. An EyeLink 1000 eye-tracking system (SR Research inc.,

9 Canada), with high spatial and temporal resolution, recorded each participant's right-eye gaze

10 location every millisecond during binocular viewing. Displays were presented on a 19-inch

11 monitor $(150 \mathrm{~Hz}$ refresh-rate, $1024 \times 768$ resolution $)$. Each picture was $10.4^{\circ} \times 7.2^{\circ}$, with a

12 distance of $3.2^{\circ}$ from its inner edge to the display screen centre.

13 At the start of the experiment, each participant completed a 9-point calibration

14 procedure. On one third of trials participants indicated, using a mouse-click, which of the

15 pictures they liked most, and on another third of trials, which they liked least. For the

16 remaining third of trials, there was no instruction and no response was required. Each display

17 was presented for $5000 \mathrm{~ms}$ and participants could look at the pictures in any order. The whole

18 experiment lasted 40 minutes.

19 Following the eye-tracking task, participants received an (unexpected) recognition

20 memory task. They were instructed to indicate, using a key press, whether they had seen the

21 picture presented in the memory test in the eye movement experiment. All 36 stimuli were 
1 included, along with 36 unseen pictures, with order of stimuli presentation counterbalanced

2 across participants. Figure 1 shows a schematic of the trial sequence.

\section{Insert Figure 1}

Results

Invalid data (including data recorded when participants moved their head or where

fixations were off-centre at the beginning of a trial by $>1^{\circ}$ ) were excluded from analysis

$(<0.5 \%$ in total). Following standard procedures, fixations shorter than $80 \mathrm{~ms}$ or longer than

$1200 \mathrm{~ms}$ were also removed (affecting $<0.5 \%$ of trials). We collected data from the interest areas (each picture in the display) and calculated the time spent looking in that area, gaze duration (GD), before participants inspected another picture. The proportion of the first fixations landing in an interest area and the number of fixations made to an interest area (from first fixation up to leaving that area) are also reported in the main text. Data were analysed using Linear Mixed-Effects Models (LMMs, Baayen, Davidson, \& Bates, 2008) using R (R Development Core Team, 2016) and the lme4 package (Bates, Mächler, Bolker, \& Walker, 2014). A maximal random effects structure was used where possible (see Barr, Levy, Scheepers, \& Tily, 2013). The MASS package (Venables \& Ripley, 2002) was used to define main effects of age group, culture, and a contrast matrix was used to assess picture type effects. Following convention, $t>1.96$ was considered statistically significant. Means and standard errors are summarised in Table 2. The statistics for Eye Movement Measures and Memory accuracy are summarised in Table 3 
2 attentional orienting or preference for one picture type compared with the others. The main

3 effect of picture type was significant, the probability of first fixation was higher for pleasant

4 pictures (36\%) followed by the the unpleasant pictures (33\%), and least for neutral (31\%)

5 pictures. No other effects were significant, $z s<1.96$. This finding indicates that all

6 participants were more likely to initially orient to the pleasant pictures. engage with all three pictures in the display, if there were any group differences in the time spent engaging with one type of stimuli compared to another (before moving the eyes to a

10 different picture) then this would indicate preferential processing for that type of stimulus for 11 one group over another. This is what we looked at next.

Gaze Duration (GD). Gaze duration is the summed duration of all fixations from the

13 first fixation within a region until a fixation is made outside that region. Participants make

14 more fixations on pictures (before moving the eyes to a different picture) when they show a

15 preference for that picture. Longer GDs indicate increased focus and engagement with a

16 stimulus, from which one can infer preferential processing of that stimulus. The main effect

17 of age was significant, GD was longer for young adults than their older counterparts. The

18 main effect of picture-type was significant, GD was longer for pleasant pictures followed by

19 unpleasant, and least for neutral pictures. The interaction between age-group and picture-type

20 for pleasant vs unpleasant was also significant. Follow-up contrasts showed both older and

21 young participants looked more at pleasant than unpleasant pictures, but, this effect was 
1 significantly larger for older adults, $b=101.92, S E=14.66$, $t=6.95$, than young adults, $b=48.40$,

$2 S E=14.65, t=3.30$. More crucially, the three-way interaction was not significant, suggesting

3 the PE is similar in both cultures. No other effects were observed, $t s<1.96$. Figure 2 panel

4 (a) shows the interaction between age-group and picture-type.

Number of first pass fixations. The number of first-pass fixations is the count of all

6 fixations within a region from the first fixation until a fixation is made outside this region.

7 For this measure, the data showed a similar pattern to the GD measure. A main effect of age

8 reflects the propensity of young adults to have more fixations than their older counterparts

9 overall. The main effect of picture type was also significant, numbers of fixations was greater

10 for pleasant pictures followed by unpleasant, and least for neutral pictures. The interaction

11 between age-group and picture-type was observed for comparisons of age-group x pleasant

12 vs unpleasant and age-group x neutral vs unpleasant. Follow-up contrasts showed both older

$13(b=.33, S E=.05, t=7.25)$ and young $(b=.16, S E=.05, t=3.46)$ adults looked more often

14 at pleasant than unpleasant pictures, but again this effect was larger for the older than the

15 young adults. Follow-up contrasts for neutral vs unpleasant revealed more fixations for

16 unpleasant than neutral pictures $(b=0.23, S E=0.04, t=5.47)$, with no difference for older

17 adults $(b=0.07, S E=0.04, t=1.66)$. Again, the three-way interaction was not significant,

18 indicating the PE to be similar in both cultures. No other effects were observed, $t<1.96$.

19 Figure 2 panel (b) shows the interaction between age-group and picture-type.

In sum, the number of fixations measure is in line with the GD measure, showing a

21 clear PE. However, the probability of first fixation shows only a main effect for pleasant 
1 pictures. This measure was not modulated by age, showing that the GD measure (the sum of

2 the fixations once a picture has been initially fixated) is a more sensitive measure of the PE in

3 the current study, and revealing that the older participants have a preference to explore the

4 pleasant pictures in more detail before moving the eyes to look at the other pictures in the

5 display.

Recognition Memory Accuracy. We tested for picture recognition accuracy by

7 calculating the proportion of pictures correctly identified as seen previously in the eye

8 movement experiment. A main effect of picture-type was significant, and showed that all

9 participants remembered pleasant pictures most, followed by neutral pictures, and

10 participants remembered unpleasant pictures the least well. A main effect also showed that

11 young adults had higher accuracy overall than older adults. An interaction between culture

12 and picture-type was found, and follow-up contrasts showed higher recognition accuracy for

13 neutral over unpleasant pictures for UK participants $(b=7.17, S E=1.79, t=4.00)$, but not China

14 participants $(b=2.26, S E=1.83, t=1.23)$. An interaction between age-group and picture-type

15 was also observed, and follow-up contrasts showed higher recognition accuracy for pleasant

16 over unpleasant pictures for older adults $(b=18.06, S E=1.57, t=11.48)$, but not young adults

$17(b=1.67, S E=1.54, t=1.08)$. Follow-up contrasts for neutral vs unpleasant revealed higher

18 recognition accuracy for neutral over unpleasant for older adults $(b=13.02, S E=1.57, t=$

19 8.28), while the young adults showed slightly higher recognition accuracy for unpleasant

20 over neutral $(b=3.17, S E=1.54, t=2.06)$. More importantly, the three-way interaction was 
1 not significant, $p>0.05$. Figure 2 panel (c) shows the interaction between age-group and

2 picture-type.

\section{Insert Figure 2}

Bayes factors (Kass \& Raftery, 1995) were also computed, using the lmBF function within the BayesFactor package (Morey \& Rouder, 2015) in R (R Core Team, 2015), with the scaling factor for g-priors set to 0.5 , and using 100,000 Monte Carlo iterations, in order to determine whether the PE is actually similar across both cultures, as suggested by the LMM analysis. Participants were specified as random factors. Following Vandekerckhove, Matzke, and Wagenmakers (2015), Bayes factors $(\mathrm{BFs})>3$ were taken to provide weak to moderate support for a model, and BFs $>10$ to provide strong support, while BFs $<1$ were taken to provide evidence against a model and in favor of the base model. Based on the LMM analysis, we constructed a denominator model (base model to which other models were compared) for each measure. Bayes factor analysis favored the denominator model over a model containing main effects of age group and picture type plus a three-way interaction between age, culture and picture type (gaze duration, $\mathrm{BF}=84128$; the number of first pass fixations, $\mathrm{BF}=32536$, memory accuracy, $\mathrm{BF}=3.15 \times 10^{12}$ ), suggesting that the age related PE is independent of culture.

Discussion

Our aim was to compare the PE in Eastern (China) and Western (UK) cultures when viewing emotional pictures. In an eye-tracking task, both older and younger adults fixated for longer on pleasant pictures, before moving on to inspect another picture in the display. 
1 However, the magnitude of this effect was significantly greater in the older adults, suggesting

2 an age difference in the preference for looking at pleasant emotional images, especially in the

3 attentional engagement stage, which is reflected by the GD and number of first pass fixation

4 measures. This effect was essentially the same across the Chinese and UK participants, and is

5 consistent with an age-related bias for positive emotional information (Carstensen et al.,

6 1999; Charles et al., 2003; Mather \& Carstensen, 2003; Isaacowitz et al., 2006, 2007) in both

7 Eastern and Western cultures.

A preference for pleasant pictures was also observed in an unexpected memory test

9 (Charles et al., 2003) given to the participants following the eye tracking study. Recognition

10 memory for pleasant over unpleasant pictures was evident for older, but not younger adults,

11 from both cultures. Older participants not only remembered more pleasant compared to

12 unpleasant pictures, but remembered more neutral compared to unpleasant pictures,

13 suggesting unpleasant pictures received the least processing (were avoided) in older adults,

14 with no such effect for young adults. Moreover, these effects were obtained even though task

15 demands required all pictures to be engaged with and processed in each trial in the eye

16 movement task. Our findings are therefore consistent with a PE in attention and memory in

17 both Eastern and Western groups.

These findings run counter to the claim that cultural differences in emotional

19 processing are underpinned by collectivist or individualistic cultural practices, with such

20 practices having been proposed to produce an absence of the PE in Eastern cultures (Fung et

21 al., 2008). This controversial claim has, however, received mixed empirical support, with 
1 some studies reporting an absence of a PE in Eastern samples (Fung et al., 2008), while

2 others provide evidence to support the existence of a PE (Kwon et al., 2009), including

3 evidence from an earlier eye-tracking study from our group which showed that older Chinese

4 participants preferentially looked at positive emotional information (Wang et al., 2015).

5 However, none of those studies has directly compared effects cross-culturally in the same

6 experiment, and the Fung et al.'s claim is based on a null effect. Consequently, the present

7 research contributes to this debate by showing that the PE is found cross-culturally, for both

8 Eastern and Western participants in the same experiment. With the present study, we show

9 consistent age differences in PE across both an eye-tracking experiment and a follow-up

10 memory experiment for China and UK participants; while Bayesian analysis support an

11 interpretation of the findings in which the PE is essentially the same across the two cultures.

12 Such findings are in line with SST theory, which posits that the perception of limited

13 future life-time is what leads to the development of a PE in older people. Moreover, the

14 theory that older adults pursue more positive related immediate goals, and younger adults

15 focus more on goals that have longer term impact appears to be cross-cultural. Thus, the PE

16 reflects an ageing effect, and the evidence from the current study suggests that this ageing

17 effect is likely to be independent of cultural emotional processing differences. In summary, by comparing the PE cross-culturally, using a novel design permitting

19 presentation of pleasant, unpleasant and neutral information in a single trial, we replicate key

20 previous findings (Isaacowitz et al., 2006; Wang et al., 2015) and show consistent age

21 differences in the PE in preferential viewing and recognition memory for both Chinese and 
1 UK participants. Our analysis show that the PE is essentially the same across Eastern and

2 Western cultures and, resonant with SST theory, the findings suggest that ageing, and not

3 culture, determines the PE. Indeed, as ageing is fundamental, irrespective of culture, an age-

4 related bias for positive information may exist across Western and Eastern cultures, and this

5 bias may even be universal.

6 
2 This work was supported by a National Natural Science Foundation of China grant

3 (81771823) to Jingxin Wang, a Chinese Scholarship Council research visit grant to Fang Xie,

4 a Recruitment to Foreign Experts award to Valerie Benson, and a 1000 Talents Visiting

5 Professorship to Kevin Paterson.

6 
Disclosure statement

No potential conflict of interest was reported by the authors. 


\section{References}

Baayen, R. H., Davidson, D. J., \& Bates, D. M. (2008). Mixed-effects modeling with crossed random effects for subjects and items. Journal of Memory and Language, 59, 390-412. http://dx.doi.org/10.1016/j.jml .2007.12.005

Barr, D. J., Levy, R., Scheepers, C., \& Tily, H. J. (2013). Random effects structure for confirmatory hypothesis testing: Keep it maximal. Journal of Memory and Language, 68, 255-278. http://dx.doi.org/10.1016/j.jml .2012.11.001

Bates, D., Mächler, M., Bolker, B., \& Walker, S. (2014). Fitting linear mixed-effects models using lme4. arXiv preprint arXiv:1406.5823. Retrieved from http://CRAN.Rproject.org/package $=$ lme 4

Carstensen, L.L., Isaacowitz, D.M., \& Charles, S.T. (1999). Taking time seriously: a theory of socioemotional selectivity. American Psychologist, 54, 165-181. doi:10.1037/0003066X.54.3.165

Charles, S.T., Reynolds, C.A., \& Gatz, M. (2001). Age-related differences and change in positive and negative affect over 23 years. Journal of Personality and Social Psychology, 80, 136-151. doi:10.1037/0022-3514.80.1.136

Charles, S.T., Mather, M., \& Carstensen, L.L. (2003). Aging and emotional memory: The forgettable nature of negative images for older adults. Journal of Experimental Psychology: General, 132, 310-324. doi : 10.1037/0096-3445.132.2.310

Chung, C., \& Lin, Z. (2012). A cross-cultural examination of the positivity effect in memory: United States vs. China. International Journal of Aging \& Human Development, 75(1), 
31-44. doi: http://dx.doi.org/10.2190/AG.75.1.d

Fung, H.H., Lu, A.Y., Goren, D., Isaacowitz, D.M., Wadlinger, H.A., \& Wilson, H.R. (2008). Age-related positivity enhancement is not universal: older Chinese look away from positive stimuli. Psychology and Aging, 23, 440-446. doi:10.1037/0882-7974.23.2.440

Hess, T.M., Auman, C., Colcombe, S.J., \& Rahhal, T.A. (2003). The impact of stereotype threat on age differences in memory performance. Journals of Gerontology, 58, 3-11.

Isaacowitz, D.M., Wadlinger, H.A., Goren, D., \& Wilson, H.R. (2006). Selective preference in visual fixation away from negative images in old age? An eye-tracking study. Psychology and Aging, 21, 40-48. doi:10.1037/0882-7974.21.1.40

Isaacowitz, D.M., Löckenhoff, C.E., Lane, R.D., Wright, R., Sechrest, L., \& Riedel, R. (2007). Age differences in recognition of emotion in lexical stimuli and facial expressions. Psychology \& Aging, 22, 147-159. doi:10.1037/0882-7974.22.1.147

Isaacowitz, D.M., Livingstone, K.M., Harris, J.A., \& Marcotte, S.L. (2015). Mobile eye tracking reveals little evidence for age differences in attentional selection for mood regulation. Emotion, 15, 151-161. doi:10.1037/emo0000037

Kass, R.E., \& Raftery, A.E. (1995). Bayes factors. Journal of the American Statistical Association, 90, 773-795.

Kwon, Y., Scheibe, S., Samanezlarkin, G.R., Tsai, J.L., \& Carstensen, L.L. (2009). Replicating the positivity effect in picture memory in Koreans: evidence for crosscultural generalizability. Psychology and Aging, 24, 748-754. doi:10.1037/a0016054 Lang, P. J., Bradley, M. M., \& Cuthbert, B. N. (2005). International Affective Picture System 
(IAPS): Digitized photographs, instruction manual and affective ratings (Technical Report A:6). Gainesville, FL: University of Florida.

Mather, M., \& Carstensen, L.L., (2003). Aging and attentional biases for emotional faces. Psychological Science, 14, 409-415.

Morey, R. D., \& Rouder, J. N. (2015). BayesFactor: Computation of Bayes factors for common designs. R package version 0.9.12-2. Retrieved from http://CRAN.Rproject.org/package=BayesFactor .

Mroczek, D.K., \& Kolarz, C.M. (1998). The effect of age on positive and negative affect: a developmental perspective on happiness. Journal of Personality and Social Psychology, 75, 1333-1349. doi:10.1037/0022-3514.75.5.1333

Nasreddine, Z.S., Phillips, N.A., Bédirian, V., Charbonneau, S., Whitehead, V., Collin, I., Cummings, J.L., \& Chertkow, H. (2005). The Montreal Cognitive Assessment, MoCA: a brief screening tool for mild cognitive impairment. Journal of the American Geriatric Society, 53, 695- 699. http:// dx.doi.org/10.1111/j.1532-5415.2005.53221

R Development Core Team (2016). R: A language and environment for statistical computing. R Foundation for Statistical Computing, Vienna, Austria. https://www.R-project.org/ Reed, A.E., \& Carstensen, L.L. (2012). The theory behind the age-related positivity effect. Frontiers in Psychology, 3, 339. doi:10.3389/fpsyg.2012.00339

Ryan, J.J., Sattler, J.M., \& Lopez S.J. (2000). Age effects on Wechsler Adult Intelligence Scale-III subtests. Archives of Clinical Neuropsychology, 15, 311-317.

Taylor, H. R. (1978). Applying new design principles to the construction of an illiterate E 
chart. American Journal of Optometry and Physiological Optics, 55, 348-351.

Tsai J.L., Knutson, B., Fung, H.H. (2006). Cultural variation in affect valuation. Journal of Personality and Social Psychology, 90, 288 -307.

Vandekerckhove, J., Matzke, D., \& Wagenmakers, E.J. (2014). Model comparison and the principle of parsimony. In J.R. Busemeyer, Z. Townsend, J. Wang, \& A. Eidels (Eds.), Oxford handbook of computational and mathematical psychology (pp. 125-144).

Oxford: Oxford University Press.

Venables, W. N., \& Ripley, B. D. (2002). Modern applied statistics with S (4th ed.). New York, NY: Springer. http://dx.doi.org/10.1007/978-0-387-21706-2

Watson, D., Clark, L., \& Tellegen, A. (1988). Development and validation of brief measures of positive and negative affect: The PANAS scales. Journal of Personality and Social Psychology, 54, 1063-1070

Wang, J., He, L., Jia, L., Tian, J., \& Benson, V. (2015). The 'positive effect' is present in older Chinese adults: Evidence from an eye tracking study. PloS ONE, 10, e0121372.

Wechsler, D. (1997). WAIS-III administration and scoring manual. San Antonio, TX:

Psychological Corporation. 
Ageing and Positive Emotional Biases

Figure legend

Figure 1 shows a schematic of the trial sequence.

Figure 2 shows the interactions between age group and picture type for Eye movement measures and recognition memory accuracy. Panel (a) shows the interaction between Age and Picture Type for GD; Panel (b) shows the interaction between Age and Picture Type for numbers of first pass fixation; Panel (c) shows the interaction between Age and Picture Type for memory accuracy. 
Ageing and Positive Emotional Biases

27

Table 1. Correlation coefficients for ratings of the valence and arousal of picture by different groups relative to rating scores for Western young adults obtained from the IAPS database.

\begin{tabular}{|c|c|c|c|}
\hline & $\begin{array}{l}\text { Young adults } \\
\text { (Eastern) }\end{array}$ & $\begin{array}{l}\text { Older adults } \\
\text { (Eastern) }\end{array}$ & $\begin{array}{l}\text { Older adults } \\
\text { (Western) }\end{array}$ \\
\hline $\begin{array}{c}\text { Valence } \\
\text { Young samples } \\
\text { (USA) }\end{array}$ & $r=.93 * * *$ & $r=.91 * * *$ & $r=.93 * * *$ \\
\hline $\begin{array}{c}\text { Arousal } \\
\text { Young samples } \\
\text { (USA) }\end{array}$ & $r=.65 * * *$ & $r=.54 * * *$ & $r=.53 * * *$ \\
\hline
\end{tabular}

Note. Significant effects are indicated with asterisks. $* * * p<.001$ 
Table 2. Means and SEs for Eye movement measures and Memory

\begin{tabular}{|c|c|c|c|c|c|c|c|}
\hline \multirow{3}{*}{ Measures } & \multicolumn{4}{|c|}{ Older } & \multicolumn{3}{|c|}{ Younger } \\
\hline & \multicolumn{4}{|c|}{ Picture type } & \multicolumn{3}{|c|}{ Picture type } \\
\hline & Culture & $\mathrm{P}$ & $\mathrm{U}$ & $\mathrm{N}$ & $\mathrm{P}$ & $\mathrm{U}$ & $\mathrm{N}$ \\
\hline The probability of & China & $.36(.01)$ & $.33(.01)$ & $.31(.01)$ & $.36(.01)$ & $.33(.01)$ & $.31(.01)$ \\
\hline \multirow[t]{2}{*}{ first fixation } & UK & $.36(.01)$ & $.33(.01)$ & $.31(.01)$ & $.37(.01)$ & $.33(.01)$ & $.30(.01)$ \\
\hline & China & $629(7)$ & $515(5)$ & $491(5)$ & $771(8)$ & $703(8)$ & $668(8)$ \\
\hline \multicolumn{8}{|l|}{ Gaze duration (GD) } \\
\hline & UK & $713(8)$ & $623(7)$ & $598(7)$ & $749(9)$ & $720(9)$ & $644(8)$ \\
\hline Number of first pass & China & $2.4(.02)$ & $2.1(.02)$ & $2.0(.02)$ & $2.8(.03)$ & $2.5(.03)$ & $2.4(.02)$ \\
\hline \multirow[t]{2}{*}{ fixations } & UK & $2.7(.03)$ & $2.4(.02)$ & $2.4(.02)$ & $2.8(.03)$ & $2.7(.03)$ & $2.4(.03)$ \\
\hline & China & $93.1(1.6)$ & $76.0(2.1)$ & $86.1(1.9)$ & $93.4(1.7)$ & $94.1(1.8)$ & $88.5(1.8)$ \\
\hline
\end{tabular}


Recognition

Memory accuracy

UK

94.8 (1.6)

$75.7(2.3)$

$91.7(2.0)$

$96.5(1.1)$

$92.6(1.4)$

$91.7(1.5)$

$(\%)$

Note. Standard Error of the means are provided in parentheses. $\mathrm{P}=$ pleasant; $\mathrm{N}=$ neutral; $\mathrm{U}=$ unpleasant 
Table 3 Linear Mixed Model Statistics for Eye Movements Measures and Memory accuracy

\begin{tabular}{|c|c|c|c|c|c|}
\hline Source of variance & & $\begin{array}{c}\text { The probability of } \\
\text { first fixation }\end{array}$ & Gaze duration & $\begin{array}{l}\text { Number of first } \\
\text { pass fixations }\end{array}$ & $\begin{array}{c}\text { Recognition Memory } \\
\text { accuracy }(\%)\end{array}$ \\
\hline \multirow[t]{3}{*}{ Intercept } & $b$ & 0.70 & 651.66 & 2.47 & 89.57 \\
\hline & $S E$ & 0.01 & 15.71 & 0.06 & 0.61 \\
\hline & $t / z$ & 66.54 & 41.47 & 43.69 & 146.76 \\
\hline \multirow[t]{3}{*}{ Age Group } & $b$ & 0.00 & 114.87 & 0.28 & 6.68 \\
\hline & $S E$ & 0.02 & 31.43 & 0.11 & 1.22 \\
\hline & $t / z$ & 0.02 & $3.66^{*}$ & $2.44 *$ & $5.47 *$ \\
\hline \multirow[t]{3}{*}{ Culture } & $b$ & 0.00 & 44.73 & 0.20 & 2.05 \\
\hline & $S E$ & 0.02 & 31.43 & 0.11 & 1.22 \\
\hline & $t / z$ & 0.08 & 1.42 & 1.79 & 1.68 \\
\hline \multicolumn{6}{|l|}{ Picture type } \\
\hline \multirow[t]{3}{*}{ P vs. N } & $b$ & 0.25 & 115.31 & 0.39 & 4.93 \\
\hline & $S E$ & 0.03 & 7.28 & 0.02 & 1.09 \\
\hline & $t / z$ & $8.76^{*}$ & $15.85^{*}$ & $16.45^{*}$ & $4.51 *$ \\
\hline U vs. N & $b$ & 0.10 & 40.18 & 0.15 & 4.88 \\
\hline
\end{tabular}




\begin{tabular}{|c|c|c|c|c|c|}
\hline & $S E$ & 0.03 & 8.94 & 0.03 & 1.09 \\
\hline & $t / z$ & $4.08^{*}$ & $4.49 *$ & $5.17 *$ & $4.47 *$ \\
\hline \multirow[t]{3}{*}{ P vs. U } & $b$ & 0.14 & 75.13 & 0.24 & 9.82 \\
\hline & $S E$ & 0.03 & 10.34 & 0.03 & 1.09 \\
\hline & $t / z$ & $5.10 *$ & $7.26^{*}$ & $7.63 *$ & $8.98^{*}$ \\
\hline \multicolumn{6}{|l|}{ Age Group * Culture } \\
\hline & $b$ & 0.00 & 108.84 & 0.25 & 0.54 \\
\hline & $S E$ & 0.04 & 62.85 & 0.23 & 2.44 \\
\hline & $t / z$ & 0.04 & 1.73 & 1.09 & 0.22 \\
\hline \multicolumn{6}{|c|}{ Age Group * Picture type } \\
\hline \multirow[t]{3}{*}{ Age Group * P vs. N } & $b$ & 0.05 & 22.03 & 0.02 & 0.20 \\
\hline & $S E$ & 0.06 & 14.55 & 0.05 & 2.19 \\
\hline & $t / z$ & 0.88 & 1.51 & 0.32 & 0.09 \\
\hline \multirow[t]{3}{*}{ Age Group * U vs. N } & $b$ & 0.02 & 31.46 & 0.16 & 16.28 \\
\hline & $S E$ & 0.05 & 17.89 & 0.06 & 2.19 \\
\hline & $t / z$ & 0.41 & 1.76 & $2.77 *$ & $7.45^{*}$ \\
\hline \multirow[t]{2}{*}{ Age Group * P vs. U } & $b$ & 0.03 & 53.48 & 0.17 & 16.48 \\
\hline & $S E$ & 0.06 & 20.69 & 0.06 & 2.19 \\
\hline
\end{tabular}




\begin{tabular}{|c|c|c|c|c|c|}
\hline & $t / z$ & 0.50 & $2.59 *$ & $2.71 *$ & $7.54 *$ \\
\hline \multicolumn{6}{|l|}{ Culture * Picture type } \\
\hline \multirow[t]{3}{*}{ Culture * P vs. $\mathrm{N}$} & $b$ & 0.05 & 8.90 & 0.01 & 1.94 \\
\hline & $S E$ & 0.06 & 14.55 & 0.05 & 2.19 \\
\hline & $t / z$ & 0.89 & 0.61 & 0.15 & 0.89 \\
\hline \multirow[t]{3}{*}{ Culture $*$ U vs. $\mathrm{N}$} & $b$ & 0.01 & 21.82 & 0.11 & 5.25 \\
\hline & $S E$ & 0.05 & 17.89 & 0.06 & 2.19 \\
\hline & $t / z$ & 0.11 & 1.22 & 1.92 & $2.40 *$ \\
\hline \multirow[t]{3}{*}{ Culture * P vs. U } & $b$ & 0.04 & 30.73 & 0.12 & 3.31 \\
\hline & $S E$ & 0.06 & 20.69 & 0.06 & 2.19 \\
\hline & $t / z$ & 0.81 & 1.49 & 1.82 & 1.52 \\
\hline \multicolumn{6}{|c|}{ Age Group * Culture *Picture type } \\
\hline \multirow[t]{3}{*}{ Age Group * Culture* P vs. N } & $b$ & 0.16 & 27.01 & 0.14 & 3.77 \\
\hline & $S E$ & 0.11 & 29.10 & 0.09 & 4.37 \\
\hline & $t / z$ & 1.39 & 0.93 & 1.47 & 0.86 \\
\hline \multirow[t]{2}{*}{ Age Group * Culture* U vs. N } & $b$ & 0.03 & 41.86 & 0.21 & 1.31 \\
\hline & $S E$ & 0.10 & 35.78 & 0.11 & 4.37 \\
\hline
\end{tabular}




\begin{tabular}{lccccc} 
& $t / z$ & 0.31 & 1.17 & 1.84 & 0.30 \\
\hline Age Group * Culture* P vs. U & $b$ & 0.12 & 14.85 & 0.07 & 2.46 \\
& $S E$ & 0.11 & 41.37 & 0.13 & 4.37 \\
\hline
\end{tabular}

Note. Significant effects are indicated with an asterisk. $\mathrm{P}=$ pleasant; $\mathrm{N}=$ neutral; $\mathrm{U}=$ unpleasant. 
Ageing and Positive Emotional Biases

Figure 1

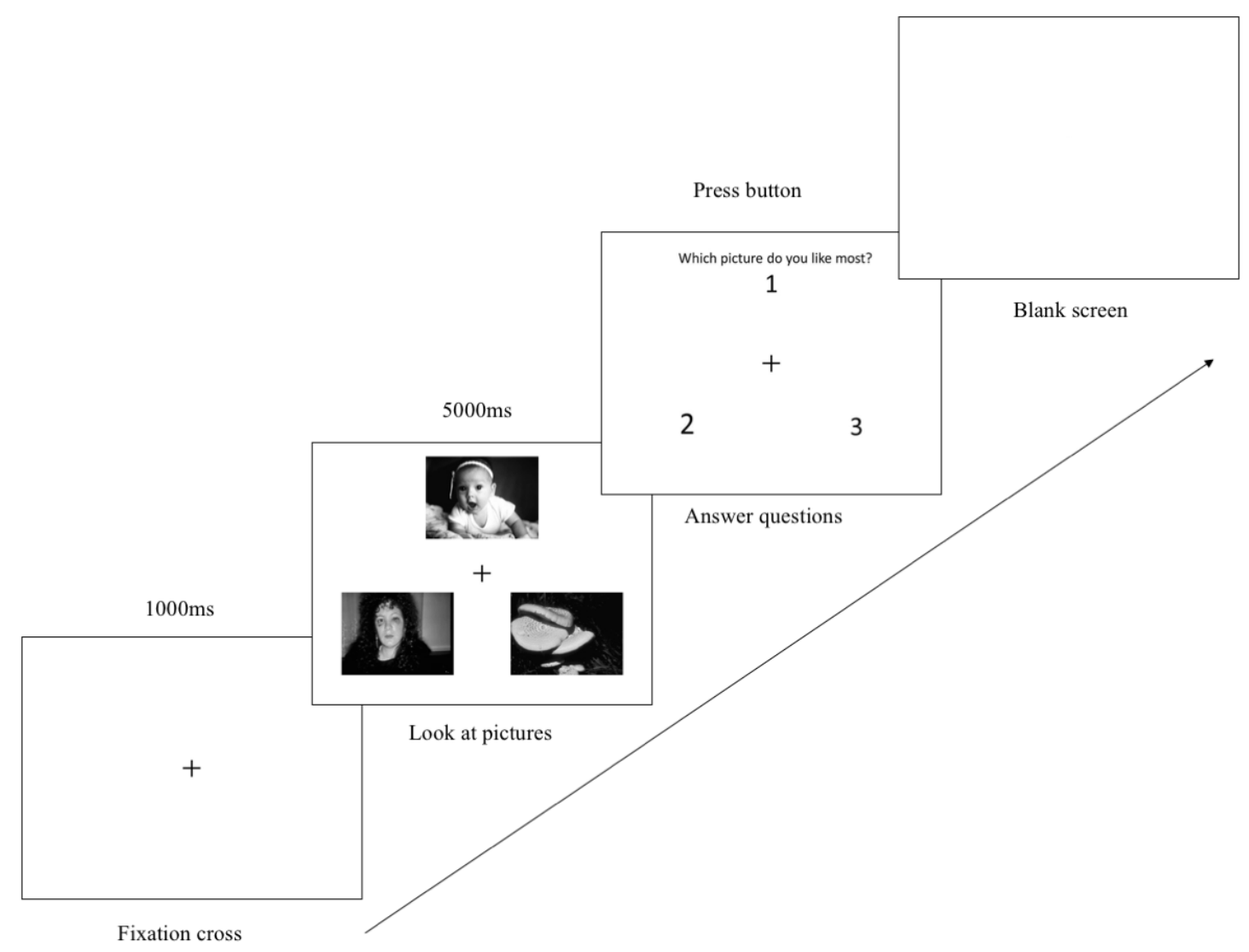


Ageing and Positive Emotional Biases

Figure 2

Panel (a)

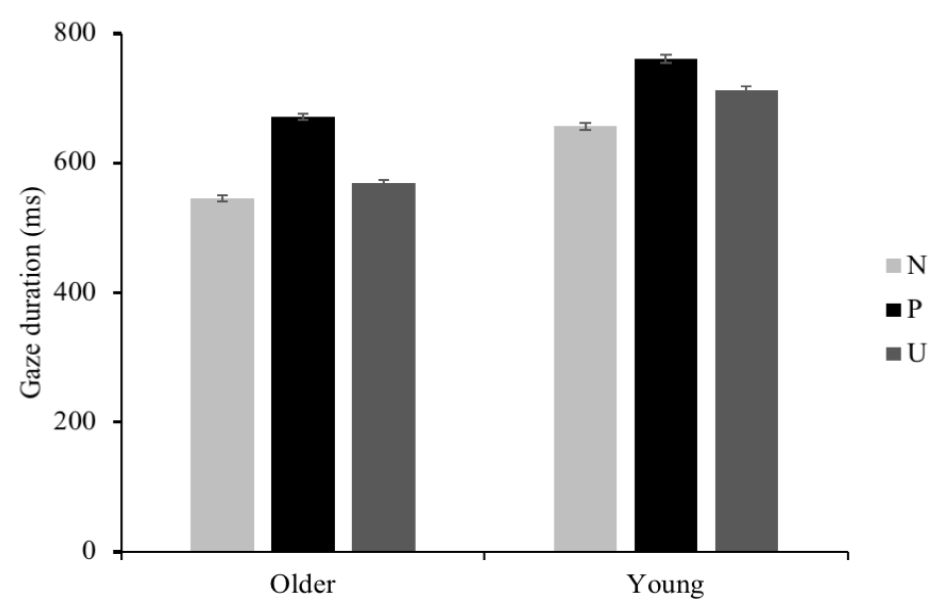

Panel (b)

Panel (c)
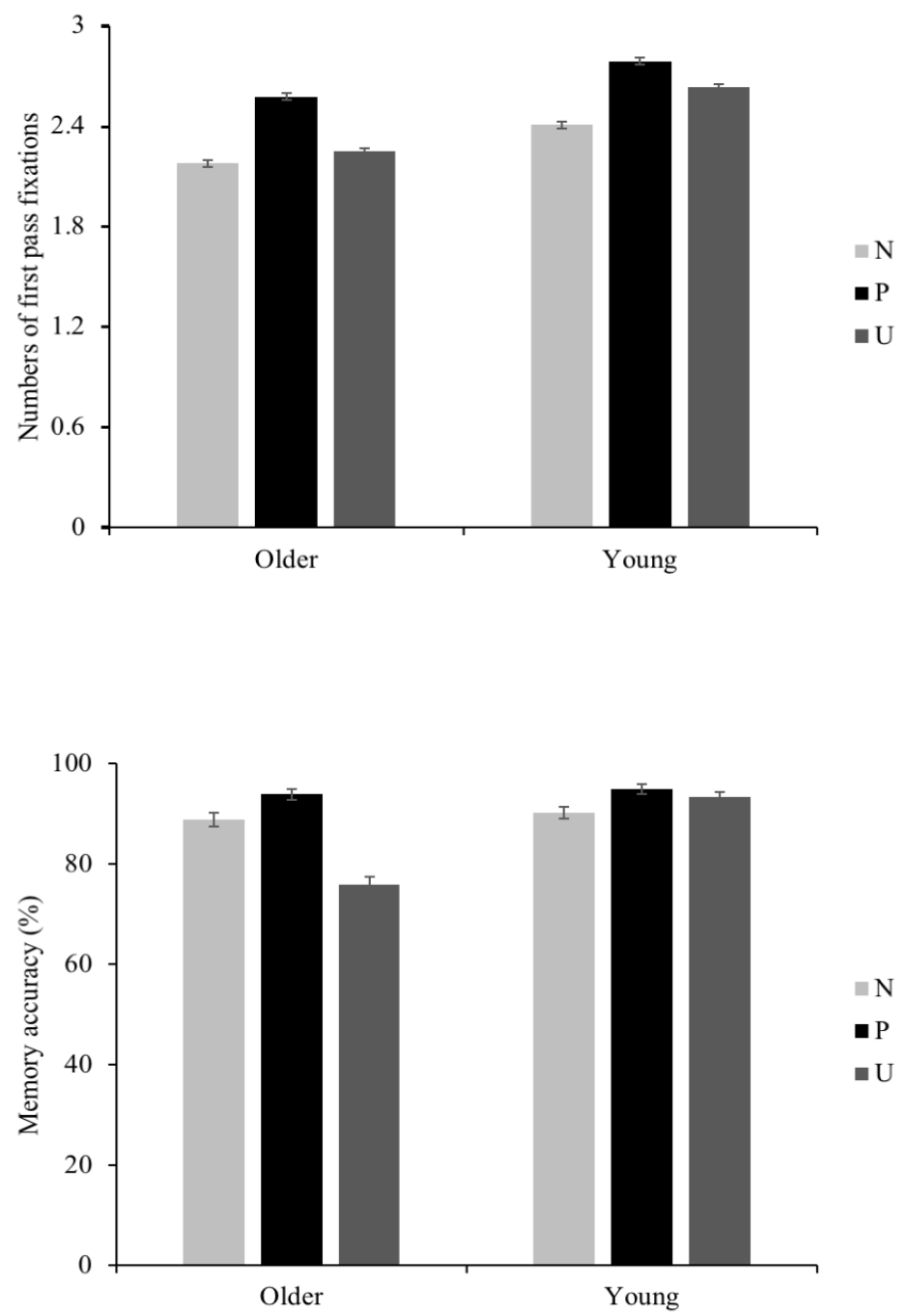